\title{
Etude électrophorétique quantitative en gélose des protéines sériques de bovins
}

\author{
par R. GIDEL \\ avec la collaboration technique de $M^{m e}$ F. LEPORT
}

La population bovine de la Haute-Volta comprend à la fois des taurins et des zébus, ainsi qu'une proportion importante de métis zébustaurins. Les taurins se rencontrent dans la moitié sud du territoire, où sévit la trypanosomiase, tandis que la partie nord est peuplée exclusivement de zébus. Les métis zébus-taurins se trouvent sur une large ligne de démarcation séparant les deux régions.

Du fait de l'existence de la trypanosomiase dans toute la partie sud du territoire, nous avons pensé qu'il pouvait être intéressant d'étudier comparativement les protéines sériques des taurins et des zébus par l'analyse électrophorétique, afin de noter si la trypanosomiase bovine amenait une modification de la répartition quantitative des protéines sériques analogue à celle constatée dans la trypanosomiase humaine.

Par suite de difficultés matérielles, notre travail n'a porté malheureusement que sur un nombre réduit d'animaux puisque nows avons dû nous limiter à l'étude de 35 sérums soif 15 sérums de taurins ef 20 sérums de zébus. Nous pensons néanmoins que les résuliats obtenus sont intéressants à signaler puisque nous avons constaté chez les taurins une modification de la répartition des protéines sériques, dans le sens de celle rencontrée chez l'homme atteint de trypanosomiase.

\section{APPAREILLAGE ET TECHNIQUE UTILISÉS}

L'appareil que nous possédions était un appareil à électrophorèse Jouan, muni d'un photo-

Rev. Elev. Méd. vét. Pays trop. 1962, 15, nº 3.

Reçu pour publication : Octobre 1962. mètre et d'un enregistreur semi-automatique en densité optique, avec la cuve spéciale pour électrophorèse en gélose. La gélose employée pour la préparation des gels était de la gélose spéciale : «agar noble» Difco. L'appareil utilisé était dépourvu de l'intégrateur de courbe et les surfaces délimitées par les courbes ont été mesurées à l'aide d'un planimètre, système Coradi.

Nous avons pratiqué la microtechnique, décrite par SCHEIDEGGER, sur lames de verre porte-objets microscopiques.

\section{Préparation des gels}

A partir de la gélose spéciale «agar noble », nous préparons une solution à 5 p. 100. La gélose ainsi préparée est débitée en petits cubes de $1 \mathrm{~cm}$ de côté environ qui sont placés dans un cristallisoir contenant de l'eau distillée. Celle-ci est renouvelée chaque jour, pendant une semaine (lavage de la gélose). On mesure ensuite le poids sec sur un échantillon de 10 grammes et on calcule ainsi la concentration en gélose. On réalise alors une solution à 2 p. 100 à laquelle on ajoute un poids équivalent de tampon de Van Sande. On obtient ainsi le milieu gélifié definitif dont la concentration en gélose titre 1 p. 100 . II ne reste plus qu'à filtrer cette solution a choud sur papier filtre dans l'autoclave ouvert. Au moment de l'emploi, il suffira de chauffer le gel de gélose au bain-marie.

\section{Préparation des plaques de gélose}

Des lames porte-objets microscopiques de $76 \times 26 \mathrm{~mm}$ sont placées sur une'surface plane obtenue en coulant un peu de gélose dans une i cuve photographique. On coule ensuite la quan- 
tité de gélose nécessaire pour obtenir sur les lames une épaisseur de gel de $1 \mathrm{~mm}$ environ. Les lames sont ensuite découpées et retirées de la cuve et un petit réservoir y est découpé au centre à l'aide d'un emporte-pièce. Dans ce «godet» sera déposée, par capillarité, une microgoutte du sérum à étudier préalablement dilué au quart. Les lames sont ensuite disposées sur la cuve à électrophorèse. La jonction entre le gel et le tampon où plongent les électrodes est assurée par de petites bandes de papierfiltre imprégnées de tampon.

\section{Electrophorèse}

Quelques minutes après le début de l'électrophorèse on dépose une microgoutte de gélose dans le réservoir central pour assurer l'étanchéité et la continuité de l'ensemble du gel

La durée de l'électrophorèse est variable. Normalemenl 45 minules suffisent pour assurer la migration des protéines. Mais par suite de variations de la tension du courant nous avons été souvent amenés à allonger le temps d'électrophorèse en fonction de la chute de tension constatée.

Immédiatement après avoir retiré les lames de la cuve à électrophorèse, on les fixe en les plongeant 30 minutes dans le fixateur (acide acétique à 5 p. 100 dans de l'alcool à 700). Après fixation, les lames sont recouvertes d'une bande de papier-filtre imprégnée d'eau distillée ef on laisse sécher quelques heures sous ventilateur. On lave ensuite les'lames soigneusement à l'eau du robinet, en enlevant les traces de papierfiltre qui ont pu adtiérer au film de gélose. Les lames sont colorées par immersion pendant 15 à 30 minutes dans la solution d'Amido-schwort7.

Après coloration, les lames sont passées rapidement à l'eau et placées dans une solution d'acide acétique à 5 p. 100 pendant $b$ à $10 \mathrm{mi-}$ nutes. Ces lames sont finalement déposées dans un cristallisoir contenant un grand volume d'eau distillée et on les y 'laisse jusqu'à ce que le fond apparaisse suffisamment clair.

\section{Tracé de la courbe ef établissement des pourcentages}

Le tracé de la courbe se fait en quelques minutes et sans difficultés à l'aide de photomètre et de l'enregistreur semi-automatique. Nous passons les lames directement au photomètre, sans décoller le film de gélose qui est extrêmement fin. La mesure des surfaces a été faite avec un planimètre Morin, système Coradi. Pour la détermination de ces surfaces, nous avons d'abord utilisé le procédé de Gauss qui consiste à déterminer une série de courbes en complétant les différents pics ou sinuosités de la courbe. Nous avons finalement adopté celui de Crook qui consiste à abaisser des perpendiculaires à la ligne de base, entre deux pics adjacents, aux points de moindre densité optique.

\section{Sérums étudiés}

Les animaux dont provenaienti les sérums étaient tous en bonne santé au moment des prises de sang. Chaque sérum a fait l'objet de deux examens sucressifs.

\section{RÉSULTATS}

Les résultats détaillés et comparés sont indiqués dans les tableaux 1,2 et 3 .

\section{DISCUSSION}

L'étude de la répartition des protéines sériques chez les bovins et chez l'homme montre une diminution marquée des albumines chez les animaux en même temps qu'une forte augmentation des globulines $\gamma$, avec; en conséquence, une inversion du rapport albumine/globuline. Ces variations sont clnssiques ef n'appellent pas de commentaires particuliers.

Beaucoup plus intéressante est: l'étude comparée des résultats obtenus chez les taurins et les zébus. L'observation des tableaux précédents montre en effet que, bien que le rapport albumine/globuline soit pratiquement identique chez les zébus ef les taurins*, on note cependant des différences notables dans la répartition des différentes protéines sériques. En' particulier, la différence la plus marquée est celle intéressant les globulines $\beta$ dont le pourcentage est fortement accru chez les taurins**. Cette aug-

* Au seuil de signification $P=0,05$ la différence entre taurins et zébus n'est pas significative.

* Au seuil de signification $P=0.05$ le pourcentage de globulines $\beta$ est significativement plus élevé chez les taurins que chez les zébus. Au même seuil de signification, les pourcentages de globulines $\alpha_{1}$ el de globulines $\gamma$ sont significativement plus élevés chez les zébus que chez les taurins. 
TABLEAU I

SERUMS DE TAURINS

\begin{tabular}{|c|c|c|c|c|c|c|c|c|}
\hline \multirow{2}{*}{$\mathrm{N}^{\circ}$} & \multicolumn{7}{|c|}{ Pourcentages } & \multirow{2}{*}{ R $\quad \frac{\mathrm{A}}{\mathrm{G}}$} \\
\hline & albumine & $\alpha 1$ & $\alpha 2$ & $\alpha 1+\alpha 2$ & $\beta$ & & $\beta+\gamma$ & \\
\hline 1 & 46,4 & 1,8 & 7,1 & 8,9 & 21,4 & 23,2 & 44,6 & 0,86 \\
\hline 2 & 39,6 & 1,7 & 5,2 & 6,9 & 19 & 34,5 & 53,5 & 0,65 \\
\hline 3 & 40,2 & 6,5 & 9,1 & 15,6 & 11,2 & 33 & 44,2 & 0,67 \\
\hline 4 & 43.5 & 1,5 & 6,5 & 8 & 25,5 & 23,5 & 48,5 & 0,77 \\
\hline 5 & 45,8 & 4,2 & 6,3 & 10,5 & 20,8 & 22,9 & 43,7 & 0,84 \\
\hline 6 & 46,5 & 2,5 & 7 & 9,5 & 22 & 22 & 44 & 0,87 \\
\hline 7 & 37,8 & 1,4 & 4 & 5,4 & 31,1 & 25,7 & 56,8 & 0,60 \\
\hline 8 & 40,9 & 4,3 & 11,8 & 16,1 & 11,8 & 31,2 & 43 & 0,83 \\
\hline 9 & 43,7 & 3,1 & 6,3 & 9,4 & - & - & 46,9 & 0,78 \\
\hline 10 & 42,6 & 4,4 & 13,2 & 17,6 & 11,8 & 27,9 & 39,7 & 0,74 \\
\hline 11 & 34,1 & 4,4 & 9,9 & 14,3 & - & - & 51,6 & 0,52 \\
\hline 12 & 44,4 & 4,5 & 7,7 & 12,2 & 28,9 & 14,5 & 43,4 & 0,80 \\
\hline 13 & 41,7 & 1,2 & 11,9 & 13,1 & 35,7 & 9,5 & 45,2 & 0,72 \\
\hline 14 & 34,9 & 4,8 & 6,3 & 11,1 & - & - & 54 & 0,53 \\
\hline 15 & 34,6 & 3,8 & 9,7 & 13,5 & - & - & 51,9 & 0,53 \\
\hline $\begin{array}{c}\text { moyen- } \\
\text { nes }\end{array}$ & 41,1 & 3,4 & 8,1 & 11,5 & 21,7 & 24,4 & 47,4 & 0,71 \\
\hline
\end{tabular}




\begin{tabular}{|c|c|c|c|c|c|c|c|c|}
\hline \multirow{3}{*}{ No } & \multicolumn{8}{|c|}{$\begin{array}{c}\text { TABLEAU II } \\
\text { SERUMS DE ZEBUS }\end{array}$} \\
\hline & \multicolumn{7}{|c|}{ Pourcentages } & \multirow{2}{*}{$\mathrm{R} \frac{\mathrm{A}}{\mathrm{G}}$} \\
\hline & albumine & $\alpha 1$ & $\alpha 2$ & $\propto 1+\propto 2$ & $\beta$ & $Y$ & $\beta+\gamma$ & \\
\hline 1 & 33,9 & 5,1 & 8,5 & 13,6 & - & - & 52,5 & 0,51 \\
\hline 2 & 44,1 & 5,9 & 8,8 & 14,7 & - & - & 41,2 & 0,79 \\
\hline 3 & 46,1 & 6,7 & 6,7 & 13,4 & 13,3 & 26,7 & 40 & 0,87 \\
\hline 4 & 51,3 & 2,6 & 10,2 & 12,8 & 10,2 & 25,7 & 35,9 & 1,05 \\
\hline 5 & 45,8 & 4,2 & 8,3 & 12,5 & - & - & 41,7 & 0,84 \\
\hline 6 & 40,9 & 4,2 & 7 & 11,2 & 7 & 40,9 & 47,9 & 0,69 \\
\hline 7 & 44,9 & 6,1 & 8,2 & 14,3 & 8,2 & 32,6 & 40,8 & 0,81 \\
\hline 8 & 31,8 & 4,4 & 8,7 & 13,1 & - & - & 55,1 & 0,46 \\
\hline 9 & 33,3 & 5,6 & 9,3 & 14,9 & 7,4 & 44,4 & 51,8 & 0,50 \\
\hline 10 & 30,4 & 5,4 & 7,1 & 11,5 & 19,6 & 37,5 & 57,1 & 0,44 \\
\hline 11 & 41,2 & 5,4 & 9,9 & 14,3 & 10,6 & 33,9 & 44,5 & 0,70 \\
\hline 12 & 33,3 & 7,6 & 9,1 & 16,7 & 15,2 & 34,8 & 50 & 0,50 \\
\hline 13 & 34,7 & 2,8 & 9,7 & 12,5 & 13,9 & 38,9 & 52,8 & 0,53 \\
\hline 14 & 39,6 & - & - & 11,5 & 15,6 & 33,3 & 48,9 & 0,65 \\
\hline 15 & 39,4 & - & - & 14,5 & 14,5 & 31,6 & 46,1 & 0,65 \\
\hline 16 & 42,8 & 7,1 & 7,1 & 14,2 & 19,1 & 23,9 & 43 & 0,75 \\
\hline 17 & 50 & 3,3 & 6,7 & 10 & 10 & 30 & 40 & 1 \\
\hline 18 & 48,3 & - & - & 15,5 & 12,1 & 24,1 & 36,2 & 0,93 \\
\hline 19 & 46,4 & - & - & 10,7 & 10,7 & 32,2 & 42,9 & 0,86 \\
\hline 20 & 47 & - & - & 12,1 & 15,2 & 25,7 & 40,9 & 0,88 \\
\hline Moyennes & 41,3 & 5,1 & 8,3 & 12,8 & 12,7 & 32,3 & 45,5 & 0,72 \\
\hline
\end{tabular}


TABIEAU III

COMPARAISON DES RESUIMATS

\begin{tabular}{|l|c|c|c|c|c|c|c|c|}
\hline Espèces & Albumines & $\alpha 1$ & $\alpha 2$ & $\alpha 1+\alpha 2$ & $\beta$ & $\gamma$ & $\beta+\gamma$ & $R \frac{A}{G}$ \\
\hline Taurins & $41,1 \pm 2,2$ & $3,4 \pm 0,8$ & $8,1 \pm 1,4$ & $11,5 \pm 1,6$ & $21,7 \pm 5$ & $24,4 \pm 4,4$ & $47,4 \pm 2,4$ & $0,71 \pm 0,06$ \\
\hline Zébus & $41,3 \pm 2,9$ & $5,1 \pm 0,8$ & $8,3 \pm 0,56$ & $13,2 \pm 0,76$ & $12,7 \pm 1,9$ & $32,3 \pm 3$ & $45,5 \pm 2,74$ & $0,72 \pm 0,08$ \\
\hline$T+Z$ & $41,2 \pm 1,8$ & $4,3 \pm 0,6$ & $8,2 \pm 0,76$ & $12,35 \pm 0,6$ & $17,2 \pm 2,4$ & $28,3 \pm 1$ & $46,5 \pm 2$ & $0,715 \pm 0,06$ \\
\hline Himains & 58,1 & 3,7 & 11,9 & 15,6 & 12,9 & 12,8 & 25,7 & 1,3 \\
\hline
\end{tabular}

mentation des globulines $\beta$ chez des animaux vivant en zone où sévit la trypanosomiase bovine, mais qui présentent un certain degré de résistance naturelle à la maladie, a-t-elle la même signification que chez l'homme? Si une réponse ne peut être donnée ici à cette question. le fait mérite néanmoins d'être constaté ef l'étude demanderait a être poursuivie, en particulier, par l'analyse immuno-électrophorétique.

Laboratoire de recherches vétérinaires de Ouagadougou

(République de Haute-Volta)

\section{SUMMARY}

\section{Electrophoretic and Quantitative Study of Bovine Serous Proteins in Agar}

By means of an electrophoresis, the author has studied the relative proportions of albumens and globulins in bovine blood to determine whether trypanosomiasis modifies the protein proportions as has been recorded in human cases. He notes an increase in the percentage of globulins $\beta$ in cattle living in areas where trypanosomiasis is enzootic but considers that further studies by immunoelectrophoresis are necessary.

\section{RESUMEN}

\section{Estudio por electroforesis cuantitativa en gelosa de las proteinas séricas de bovinos}

El autor examina por medio de la electroforesis las proporciones relativas de las albúminas y de las globulinas en la sangre de bovinos para determinar si la tripanosomiasis provoca en ellos una modificación en la repartición de las proteinas análoga a la comprobada en la tripanosomiasis humana. Comprueba el aumento del porcentaje de las globulinas 3 en los animales que viven en zona endémica tripanosómica y considera que sería necesario proseguir los estudios por inmuno-electroforesis.

\section{BIBLIOGRAPHIE}

GRABAR (P.) et BURTIN (P.). - L'analyse immuno-électrophorétique ef ses applications aux liquides biologiques humains. Mosson et Cie Editeurs, Poris 1960.

LABOUCHE (C.). - Méthode d'appréciation de la séparation des fractions obtenues par micro-électrophorèse en milieu liquide. Ann. Inst. Pasteur 1962, $102: 556,60$
LABOUCHE (C.). - Méthode mathématique d'interprétation quantitative des électrophorèses. Ann. Inst. Pasteur 1962, 102 : b61, 66.

NICOLI (J.). BERGOT (J.) et DEMARCHI (J.). Etude des protéines sériques au cours de la Irypanosomiase humaine africaine. Ann. Inst. Posteur 1961, 101 : 596, 610. 